\title{
Comparison of two models of inflammatory bowel disease in rats
}

\author{
Cristina S. Catana* ${ }^{* 1, A, B, D}$, Cristian Magdas ${ }^{* 2, A, B, D}$, Flaviu A. Tabaran ${ }^{3, B-D}$, Elena C. Crăciun ${ }^{4, B, C}$, Georgiana Deak ${ }^{2, B}$,

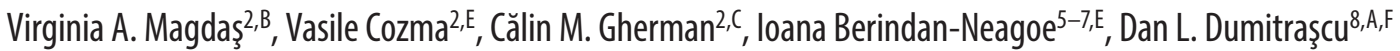 \\ ${ }^{1}$ Department of Medical Biochemistry, "Iuliu Haţieganu" University of Medicine and Pharmacy, Cluj-Napoca, Romania \\ 2 Department of Parasitology and Parasitic Diseases, University of Agricultural Sciences and Veterinary Medicine, Cluj-Napoca, Romania \\ ${ }^{3}$ Department of Anatomic Pathology, Necropsy and Forensic Medicine, University of Agricultural Sciences and Veterinary Medicine, Cluj-Napoca, Romania \\ ${ }^{4}$ Department of Pharmaceutical Biochemistry and Clinical Laboratory, "Iuliu Haţieganu“ University of Medicine and Pharmacy, Cluj-Napoca, Romania \\ ${ }^{5}$ Research Center for Functional Genomics, Biomedicine and Translational Medicine, "Iuliu Haţieganu“ University of Medicine and Pharmacy, Cluj-Napoca, Romania \\ ${ }^{6}$ Medfuture Research Center for Advanced Medicine, Cluj-Napoca, Romania \\ 7 Department of Functional Genomics and Experimental Pathology, The Oncology Institute "Prof. Dr. Ion Chiricuta", Cluj-Napoca, Romania \\ ${ }^{8} 2^{\text {nd }}$ Medical Department, "Iuliu Haţieganu" University of Medicine and Pharmacy, Cluj-Napoca, Romania \\ A - research concept and design; $\mathrm{B}$ - collection and/or assembly of data; $\mathrm{C}$ - data analysis and interpretation; \\ $\mathrm{D}$ - writing the article; $\mathrm{E}$ - critical revision of the article; $\mathrm{F}$ - final approval of the article
}

Address for correspondence

Flaviu Alexandru Tabaran

E-mail:alexandru.tabaran@usamvcluj.ro

\section{Funding sources}

None declared

\section{Conflict of interest}

None declared

* The first 2 authors contributed equally to the work.

Received on September 26, 2016

Reviewed on January 9, 2017

Accepted on February 23, 2017

\begin{abstract}
Background. There is a need for experimental animal models for inflammatory bowel diseases (IBD), but no proposed model has been unanimously accepted.

Objectives. The aim of this study was to develop 2 affordable models of IBD in rats and to compare them.

Material and methods. We produced IBD in rats using either dextran sodium sulfate (DSS) or 2, 4, 6-trinitrobenzene sulfonic acid (TNBS). The requirements for experimental models were: a predictable clinical course, histopathology and inflammation similar to human ulcerative colitis (UC) and Crohn's disease (CD). The effect of acute administration of DSS and TNBS on oxidative stress (as measured by the assessment of glutathione peroxidase - GPX) was verified. The activity of whole blood GPx was measured using a commercially available Randox kit (Crumlin, UK).

Results. The administration of DSS increased GPx activity compared to the control and TNBS-treated groups, but not to a statistically significant degree. Histological examination of the colonic mucosa following the administration of DSS showed multifocal erosions with minimal to mild inflammatory infiltrate, mainly by polymorphonuclear cells (PMN), lymphocytes and plasma cells. For TNBS-induced colitis, the histological changes manifested as multifocal areas of ulcerative colitis with mild to severe inflammatory infiltrate. Whole blood GPx values displayed a direct dependence on the chemical agent used. Our results show a correlation between histopathology, proinflammatory state and oxidative stress.
\end{abstract}

Conclusions. The experimental DSS- or TNBS-induced bowel inflammation used in this study corresponds to human IBD and is reproducible with characteristics indicative of acute inflammation in the case of the protocols mentioned.

Key words: animal model, colitis, dextran sodium sulfate, 2, 4, 6-trinitrobenzene sulfonic acid, inflammatory bowel disease

DOI

10.17219/acem/69134

Copyright

Copyright by Author(s)

This is an article distributed under the terms of the

Creative Commons Attribution Non-Commercial License

(http://creativecommons.org/licenses/by-nc-nd/4.0/) 


\section{Introduction}

Animal models can be a valuable tool in understanding complex diseases such as ulcerative colitis (UC) and Crohn's disease (CD). Progress in the research for understanding the mucosal inflammation-immune balance of inflammatory bowel disease (IBD) has been delayed by a lack of adequate experimental models. No single IBD model captures the diversity of this human disease, but each of them provides valuable insight into one main feature of IBD or another, and together they contribute to the foundation of a largely accepted set of pathogenetic principles which enhance the current therapeutic approaches to CD and UC. ${ }^{1}$ An "IBD integrom" concept offers a solution for a better understanding of the 4 components of pathogenesis, namely, genetic information (the genome); the surrounding environment (the exposome); the gut microbiota variations (the microbiome); and the intestinal immune reactivity (the immunome). ${ }^{2}$ Although the immunome is the primary effector arm of inflammation in CD and UC, having been well characterized through the IL-17/ IL-23 axis, other pathogenic processes could be implicated in the first stage of the disease. ${ }^{3,4}$ Intestinal cells die by necrosis and release many molecules which are collectively named damage-associated molecular patterns (DAMPs) - independent of pathogen-associated molecular patterns (PAMPs) - leading to traditional microbial inflammation. ${ }^{2}$ The release of DAMPs is intrinsic to IBD; these biological products trigger sterile inflammation in IBD mucosa affected by bleeding or ulcer formation. ${ }^{5}$ Moreover, there is a hypothesis that chronic inflammation due to IBD could be a combination of traditional and sterile inflammations which is known as unresolving inflammation. ${ }^{5}$ In addition, another process with contrasting results in IBD animal models is the inflammasome pathway mediated by caspase- 1 in 2,4,6-trinitrobenzene sulfonic acid-induced colitis. ${ }^{6,7}$

A variety of agents could be used as inducers of colitis: acetic acid, formalin, indomethacin, carrageenan, or immune complexes. ${ }^{8,9}$ The 2 most widely used IBD inducers in rodents are 2, 4, 6-trinitrobenzene sulfonic acid (TNBS) - ethanol administered as an enema - and dextran sulfate sodium (DSS) - often administered orally (although it can also be administered rectally) - which evoke colitis and immune inflammatory responses. ${ }^{10-12}$

The sulfated polysaccharide of DSS is directly toxic to the colonic epithelium. Thus, the short-term addition of DSS to drinking water leads to a very reproducible acute colonic inflammation as well as to a useful model for a better understanding of UC innate immune mechanisms. ${ }^{1,13}$

Intrarectal administration of a TNBS haptenating agent allows the initiation of a transmural colitis which mimics human CD and is useful for cytokine release patterns, effective immunotherapy and for the exploration of mucosal homeostasis. ${ }^{1,14}$ Ample debate still exists as to whether reactive oxygen species (ROS) are involved in the pathogenesis of tissue lesions or are endogenously produced as a consequence of damaged cells. Moreover, in contrast with normal mucosa, significantly elevated concentrations of ROS are found in the actively inflamed mucosa of patients. ${ }^{15}$ Extracellular glutathione peroxidase (E-GPx) is a selenoenzyme that reduces organic peroxides, and hydrogen peroxide. ${ }^{16} \mathrm{GPx}$ activity in humans is attributable to E-GPx. The gastrointestinal (GI) tract also produces and secretes E-GPx into the extracellular environment.

Animal models of IBD remain essential to the proper understanding of the histopathological shift in the GI tract and also play a key role in the development of novel antidotes for IBD. $1,17,18$

The aim of the study was to reproduce 2 models of IBD in rats in order to evaluate GPx activity during GI tract inflammation and to characterize the morphological changes occurring in the colonic wall. The DSS model could be valuable for the study of human UC, while the TNBS model is important for human CD.

\section{Material and methods}

In this experiment, 15 male Wistar rats weighing 200-290 g were used, in accordance with Directive 2010/63/EU of the European Parliament and the Council on the Protection of Animals used for Scientific Purposes. The animals were obtained from the Laboratory Animals Biobase of the University of Agricultural Sciences and Veterinary Medicine from Cluj-Napoca, Romania. They were maintained in a restricted access room and were housed in plastic cages under standard laboratory conditions (room temperature of $22^{\circ} \mathrm{C}$, humidity of $50-60 \%$, with a controlled 12-h light/dark cycle). They had free access to standard laboratory rodent formula pellets.

The rats were randomized into 3 groups, with 5 rats in each one. Group 1 received tap water; Group 2 received 5\% DSS (MW 5000 Da, Sigma, St. Louis, USA) in their drinking water for 7 days; Group 3 intrarectally received a single dose of $100 \mathrm{mg} / \mathrm{kg}$ of TNBS (1 M, $293.17 \mathrm{mg} / \mathrm{mL}$, product No. 92822, Fluka, Buchs, Switzerland) diluted in $50 \%$ ethanol to a concentration of $31.25 \mathrm{mg} / \mathrm{mL}$, and had free access to tap water. The total volume instilled varied between $0.64-0.8 \mathrm{~mL}$ according to the rats' weight. Under anesthesia $(10 \mathrm{mg} / \mathrm{kg}$ of xylazine and $100 \mathrm{mg} / \mathrm{kg}$ of ketamine, $1 \mathrm{M}$ ), the TNBS was instilled into the colon lumen via a polyethylene catheter (3-millimeter outer diameter) fitted onto a 1-milliliter syringe, introduced so that the tip was approx. $10 \mathrm{~cm}$ proximal to the anus. After instillation, the rats were held with the head down for $1 \mathrm{~min}$ to prevent TNBS from leaking out, and they were maintained in a head-down position at $45^{\circ}$ until they recovered from the anesthesia. 
The animals were observed daily and checked for fecal consistency. Also, water consumption and weight changes were monitored. On day 8 of the experiment, under deep anesthesia, blood samples were collected by cardiac puncture and all the animals were euthanized. Laparotomy was performed and the colon was removed as a whole and placed in a Petri dish containing a saline solution. The colon length was measured; the total length was calculated from the cecocolic junction to the rectum at the synphisic bone. Then the colon was opened along the mesenteric side and the luminal colonic surface was gently washed with an iso-osmotic saline solution to remove residual luminal content; then it was weighed. It was next immersed in a $10 \%$ neutral buffered formalin solution (Chempur, cat. No. 200-001-8, Piekary Śląskie, Poland) for $48 \mathrm{~h}$, then trimmed into 3 equal segments (representing the proximal, middle and distal colon) and processed for routine light microscopy according to standard procedures.

\section{Histopathology}

The samples were dehydrated in ethanol baths with ascending concentrations, cleared in xylene and embedded in low-melting-point paraffin wax following routine laboratory protocol. ${ }^{19}$ Multiple 4-micron sections were cut from the resulting paraffin blocks and stained with hematoxylin and eosin (H\&E). Microscopic images were captured with an Olympus BX41 optical microscope coupled with an Olympus UC30 digital camera (Olympus, Hamburg, Germany). Finally, the images were processed by Stream Basic software (Olympus Soft Imaging Solution $\mathrm{GmbH}$, Münster, Germany).

\section{Histological semiquantitative analysis}

The severity of induced colonic inflammation was graded morphologically using a semiquantitative scale by the same histopathologist in a single-blind fashion. We employed the histological scoring system previously described by Rachmilewitz for grading the severity of DSS- and TNBSinduced colitis in rodents. ${ }^{20}$ This grading system considers the following 5 structural parameters scored on a scale of 0 to 4: depth and extent of the ulcer, presence of inflammation, extent of inflammation, and location of fibrosis (Table 1).

\section{GPx activity assay}

GPx activity was measured using a RANSEL kit (Randox Laboratories LTD., cat. No. RS504, Crumlin, UK) at $37^{\circ} \mathrm{C}$ on a Cobas Mira Plus (Roche, Basel, Switzerland) analyzer at $340 \mathrm{~nm}$. The assay is based on the previously described method. $^{21}$

\section{Hemoglobin concentration assay}

The hemoglobin $(\mathrm{Hb})$ concentration was determined by the Drabkin method. ${ }^{22}$

\section{Statistical methods}

Descriptive and inferential statistics were used in our study. For the statistical analysis of data, R v. 3.2.4 (A Language and Environment for Statistical Computing, R Core Team, R Foundation for Statistical Computing, Vienna, Austria) and STATISTICA v. 6 (StatSoft, Tulsa, USA) software was used. Student's t-test was used and the equality of variances was previously tested by Levene's test. Because multiple groups were compared, the significance level $(\alpha)$ chosen for all independent t-tests was equal to 0.02 .

To evaluate the differences in repeated measurements of body weight, we used a paired t-test. Because there were 7 occasions, the significance level was adjusted after a Bonferroni correction, and it was equal to 0.01 . The results of the paired t-test were considered significant if $\mathrm{p} \leq 0.01$.

In order to evaluate the colon length differences in the 3 groups, the Kruskal-Wallis and Mann-Whitney tests were used. Because of the multiple comparisons, the significance level was adjusted after a Bonferroni correction, and it was equal to 0.02 . The statistical significance was set at $\mathrm{p} \leq 0.02$.

The Pearson correlation coefficient ( $r$ ) was also used to study the degree of correlation between the 2 quantitative variables.

All animal care and experimental protocols used in this study were approved by the Ethics Committee of "Iuliu Hațieganu” University, Cluj-Napoca, Romania (Ethics Committee approval No. 74/20.02.2014). All applicable international, national and/or institutional guidelines for the care and use of animals were followed.

Table 1. Criteria for scoring the histological lesions

\begin{tabular}{|c|c|c|c|c|c|}
\hline Score & Depth of the ulcer & Extent of the ulcer & Presence of inflammation & Extent of inflammation & Location of fibrosis \\
\hline 0 & absence of ulcer & absence of ulcer & absence of inflammation & absence of inflammation & absence of fibrosis \\
\hline 1 & mucosal involvement & punctate & minimal & mucosal & mucosa only \\
\hline 2 & $\begin{array}{l}\text { mucosal and submucosal } \\
\text { involvement }\end{array}$ & minimal & mild & $\begin{array}{l}\text { mucosal/submucosal } \\
\text { involvement }\end{array}$ & mucosa submucosa \\
\hline 3 & $\begin{array}{c}\text { penetration } \\
\text { of muscularis propria }\end{array}$ & moderate & moderate & $\begin{array}{c}\text { mucosal/submucosal and } \\
\text { muscle involvement }\end{array}$ & including muscle layer \\
\hline 4 & $\begin{array}{c}\text { penetration } \\
\text { of muscularis propria }\end{array}$ & widespread & severe & full thickness involvement & full thickness fibrosis \\
\hline
\end{tabular}




\section{Results}

After DSS or TNBS inoculation, the rats showed differences in colon weight/length, Hb concentration and GPx activity (Table 2). In the DSS and TNBS rat models, the whole blood GPx activity was higher compared to the control rats (in accordance with those of other studies), but not statistically significantly higher in the TNBSinoculated rats (Table 1). ${ }^{15,23}$

\section{Comparisons of clinical parameters among the study groups}

The Student's t-test for the global comparison of the control and DSS-treated rats indicated statistically significant differences in $\mathrm{Hb}$ levels in the 2 groups ( $\mathrm{t}$-test assuming unequal variances, $\mathrm{t}=-2.86 ; \mathrm{df}=8 ; \mathrm{p}=0.02)$. The mean level of $\mathrm{Hb}$ concentration was higher (14.32 g/dL) among the DSS-treated group compared to the controls $(12.58 \mathrm{~g} / \mathrm{dL})$. The mean level of $\mathrm{Hb}$ concentration in the DSS-treated group (14.32 g/dL) was significantly higher $(\mathrm{t}=4.846$; $\mathrm{df}=8 ; \mathrm{p}=0.001<0.02)$ compared to the TNBS group
(11.89 g/dL). Mean Hb levels were lower (11.89 g/dL), but not to a statistically significant degree, in the TNBS-treated group compared with controls $(12.58 \mathrm{~g} / \mathrm{dL})$.

The mean colon weight was significantly different $(\mathrm{t}=-5.25 ; \mathrm{df}=8 ; \mathrm{p}=0.001<0.02)$ in the control rats (2.38 g) compared to Group 3 (3.87 g). Mean colon weight values were significantly different $(\mathrm{t}=-4.61$; $\mathrm{df}=8$; $\mathrm{p}=0.002<0.02)$ in the TNBS-treated group $(3.87 \mathrm{~g})$ compared to the DSS-treated group (2.52 g).

Next, the differences in colon length in the 3 groups were established. The Kruskal-Wallis test indicated statistically significant differences among the 3 studied groups $\left(x^{2}=10.25 ; \mathrm{df}=2 ; \mathrm{p}=0.006<0.05\right)$. These statistically significant differences between the colon length $(\mathrm{cm})$ values in Groups 1 and 3 were confirmed by a post-test analysis (Mann-Whitney test; $\mathrm{p}=0.019<0.02$ ). We observed lower mean colon length levels in the TNBS-treated group compared to the controls (mean rank $=3.40$ vs 7.60). Other statistically significant differences between the colon length $(\mathrm{cm})$ values in the TNBS-treated group compared to the DSS-treated group (mean rank = 3 vs 8 ) were identified and validated by the Mann-Whitney test $(\mathrm{p}=0.007<0.02)$.

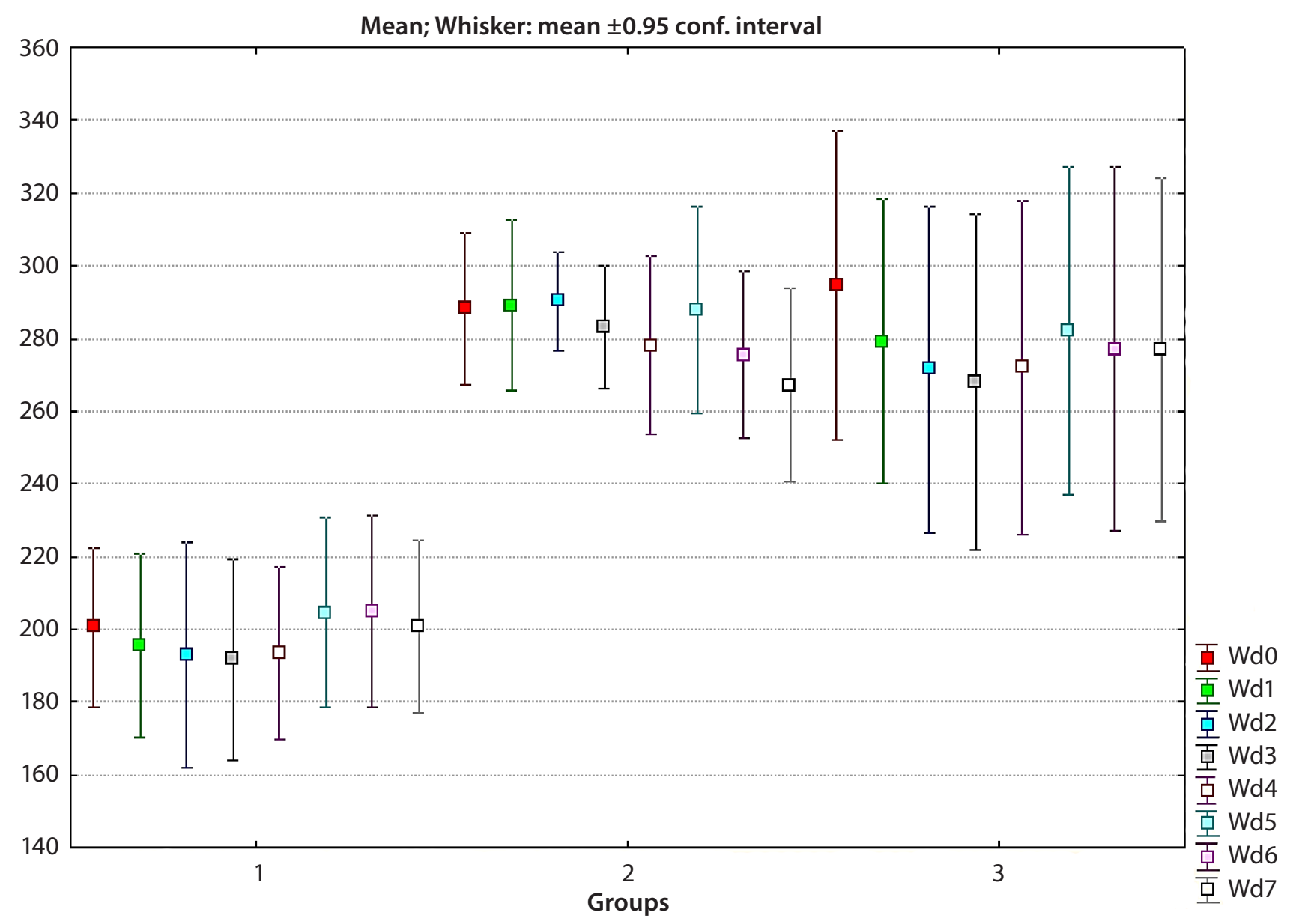

Fig. 1. Total weight evolution in studied groups over 1 week

1 - control group; 2 - DSS-treated group; 3 - TNBS-treated group. 
We also established a good negative linear correlation between colon weight and colon length $(r=-0.54$; $\mathrm{p}=0.038<0.05)$.

There were no statistically significant differences in GPx activity between the control group and the DSS group $(\mathrm{t}=-0.53 ; \mathrm{df}=8 ; \mathrm{p}=0.612)$, between the controls and the TNBS-treated group $(\mathrm{t}=0.96 ; \mathrm{df}=8 ; \mathrm{p}=0.364)$, or between DSS- and TNBS-treated groups $(\mathrm{t}=1.87$; $\mathrm{df}=8$; $\mathrm{p}=0.099$ ).

In the TNBS-treated group, significant differences in body weight were found between the initial level (Wd0) and day 1 (Wd1) (paired-sample t-test, $\mathrm{t}=7.55$; $\mathrm{df}=4$;
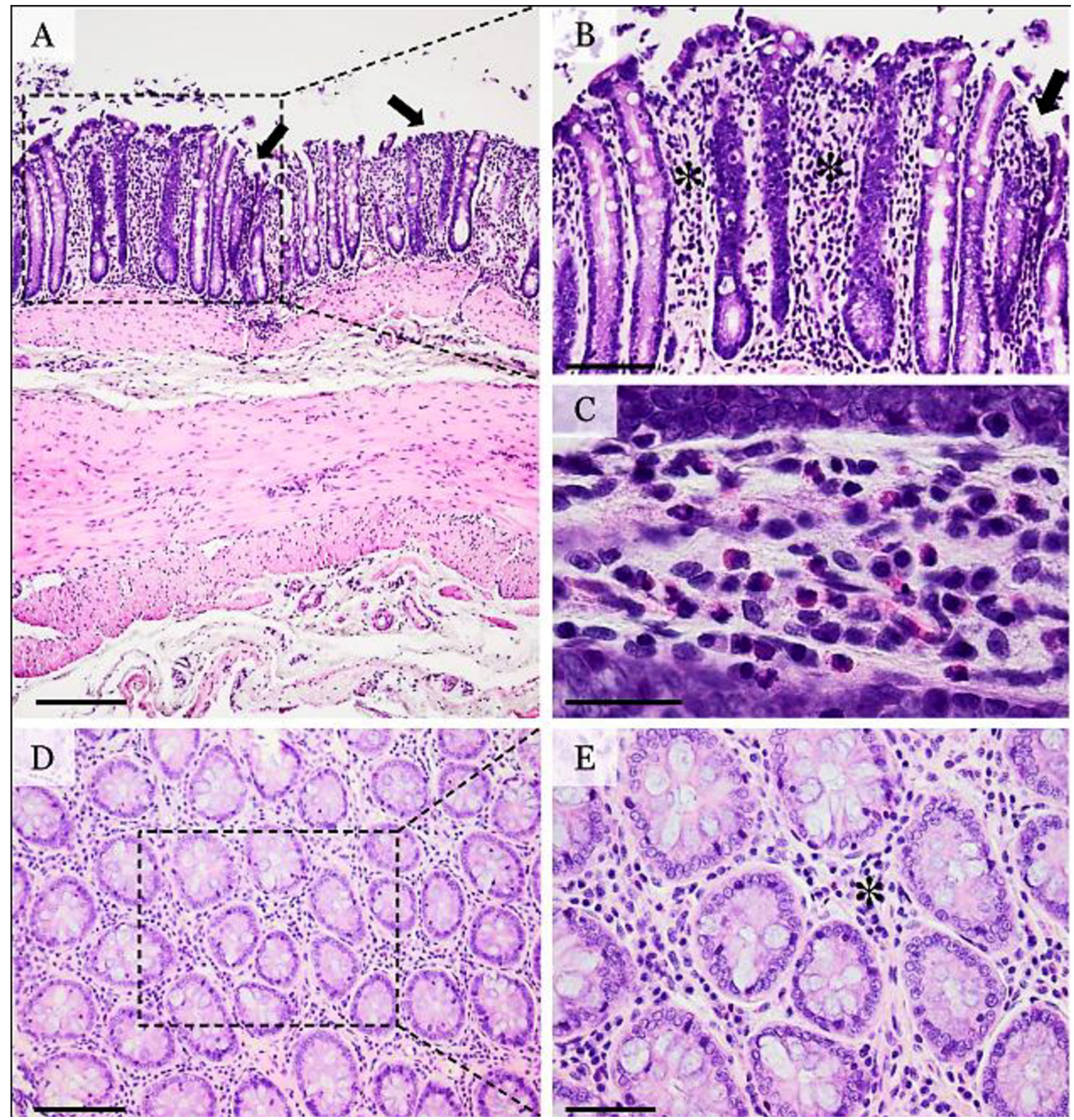

Fig. 2. Histopathological micrographs of DSS-induced colitis

\section{A - general view of the colon, presenting multiple superficial erosions (arrows) and minimal inflammatory infiltrate located in the lamina propria;}

$B$ - detail of the delineated area from image A, presenting superficial erosions (arrow) and discrete edema accompanied by mixed inflammatory infiltrate in the lamina propria; $\mathrm{C}$ - detail of the lamina propria with mixed inflammatory infiltrate represented by polymorphonuclear cells (PMN), lymphocytes and plasma cells; D - horizontal cross-section through intestinal mucosa presenting the interstitial inflammatory infiltrate and minimal fibrosis; E - detail of the delineated area from image D proves discreet interstitial fibrosis and mild inflammatory infiltration with PMN and mononuclear cells; H\&E stain, OBX-4 for image A (scale bar = $400 \mu \mathrm{m}$ ), OBX-20 for images B and D (scale bar = $80 \mu \mathrm{m}$ ), OBX-100 for image C (scale bar = $50 \mu \mathrm{m}$ ), and OBX-40 for image E (scale bar $=40 \mu \mathrm{m})$. 
$\mathrm{p}=0.002<0.01)$. The mean body weight value was significantly lower in the second evaluation (279.2 g) compared to the initial one (294.6 g).

Furthermore, in the TNBS-treated group there were also significant differences between Wd0 and Wd2 (pairedsamples t-test, $\mathrm{t}=14 ; \mathrm{df}=4 ; \mathrm{p}=0.0001<0.01)$. We observed a lower level of Wd2 (271.60 g) compared to Wd0 $(294.60 \mathrm{~g})$. The findings are the same for the $3^{\text {rd }}$ and $4^{\text {th }}$. day body weight measurements (Fig. 1).

\section{Macroscopic findings}

After the 7-day cycle of DSS administration, the mucosa presented focal congestion and superficial erosions in the middle and distal colon.

At 7 days after TNBS inoculation, we observed diffuse congestion, deep ulcers (single or multiple) - located in the middle and distal colon - multifocal ulcerative colitis, and ulceration areas coated by an adherent fibrinous material. The colon lymph nodes were congested and edematous.

\section{Histological findings}

Following the administration of DSS, the colonic mucosa showed multifocal ulcerative colitis-like lesions which tended to occur more in the posterior part of the colon

Table 2. Colon weight, colon length, hemoglobin concentration, and GPX activity in study groups

\begin{tabular}{|c|c|c|}
\hline Parameter & Group & Mean \pm SD \\
\hline \multirow{3}{*}{$\begin{array}{l}\text { Colon weight } \\
\text { [g] }\end{array}$} & 1 & $2.38 \pm 0.44$ \\
\hline & 2 & $2.51 \pm 0.47$ \\
\hline & 3 & $3.87 \pm 0.46$ \\
\hline \multirow{3}{*}{$\begin{array}{l}\text { Colon length } \\
{[\mathrm{cm}]}\end{array}$} & 1 & $22.8 \pm 1.48$ \\
\hline & 2 & $24.8 \pm 1.48$ \\
\hline & 3 & $20.80 \pm 0.44$ \\
\hline \multirow{3}{*}{$\begin{array}{l}\mathrm{Hg} \\
{[\mathrm{g} / \mathrm{dL}]}\end{array}$} & 1 & $12.58 \pm 1.12$ \\
\hline & 2 & $14.32 \pm 0.76$ \\
\hline & 3 & $11.89 \pm 0.82$ \\
\hline \multirow{3}{*}{$\begin{array}{l}\text { GPx } \\
{[\mathrm{U} / \mathrm{gHb}]}\end{array}$} & 1 & $969.38 \pm 152.06$ \\
\hline & 2 & $1013.11 \pm 105.76$ \\
\hline & 3 & $890.78 \pm 101.25$ \\
\hline
\end{tabular}

1 - control; 2 - dextran sodium sulfate; 3 - 2, 4, 6-trinitrobenzene sulfonic acid; $\mathrm{SD}$ - standard deviation; GPX - glutathione peroxidase.
(Fig. 2). The mucosal erosions were superficial, associated with discrete hyperemia and edema of the lamina propria. The inflammatory infiltrate was diffusely distributed in the mucosa, without any apparent connection to the ulcerative foci. The inflammatory population was discreetly present, being composed by a mixture of polymorphonuclear cells, lymphocytes and plasmocytes. Also, discreet interstitial fibrosis and crypt dilatation were observed.

For the TNBS-induced colitis, the histological changes presented as multifocal areas of ulcerative colitis covered by a mixture of cellular debris, fibrin, blood, and polymorphonuclear cells. The ulcerative lesions were deep, involving the mucosa and submucosa of the colon. The ulcers and associated inflammation frequently displayed a transmural pattern, inducing an overall thickening of the colonic wall. Severe villous atrophy with crypt distortion, polymorphonuclear cells and rare mononuclear leukocytes were observed to infiltrate the lamina propria, the muscularis mucosae and the submucosa (Fig. 3). Marked fibrosis of the mucosa, with disruption of the muscularis mucosae and extension through the submucosa, accompany the ulcerative process.

Statistically significant differences in the histological score were found. The mean values of histological scores and also the total score were higher in the TNBS group than in the DSS group (Table 3).

\section{Discussion}

Using DSS, a polyanionic derivative of dextran, the induced colonic inflammation starts distally after around 5 days and the lesions are confined to the colonic mucosa. DSS has a direct effect on the inner mucus layer and allows bacteria to penetrate it before any signs of inflammation are observed. ${ }^{18}$

DSS colitis is used to explore the role of inflammasome stimulation and Th-17 responses, or dectin receptors and Toll-like-receptor-initiated immune mechanisms. ${ }^{24-26}$

Also, DSS models could serve for the activation of epithelial TLR4 and releasing factors - which have positive effects and reduce the severity of DSS colonic inflammation - as well as for numerous intracellular signaling pathways. ${ }^{27,28}$ The DSS-induced colitis in rodents quantifies the process of colonic cancer in relation to IBD. ${ }^{29,30}$ Using DSS to induce colitis in Wistar rats, Kishimoto et al. mentioned that it was histologically similar to active

Table 3. Comparison of histological scores between the TNBS- and DSS-treated rat groups

\begin{tabular}{|l|c|c|c|c|c|c|}
\multirow{2}{*}{ Criterion } & \multicolumn{5}{|c}{ Mean \pm SD } \\
\cline { 2 - 6 } & depth of the ulcer & extent of the ulcer & $\begin{array}{c}\text { presence } \\
\text { of inflammation }\end{array}$ & $\begin{array}{c}\text { extent } \\
\text { of inflammation }\end{array}$ & location of fibrosis \\
\hline DSS & 1 & $1.6 \pm 0.89$ & $1.4 \pm 0.54$ & 1 & $0.6 \pm 0.54$ \\
TNBS & $2.2 \pm 0.83$ & $2.6 \pm 0.54$ & $2.6 \pm 0.54$ & $3.2 \pm 0.44$ & 2 \\
\hline p-value & 0.048 & 0.143 & 0.048 & 0.008 & 0.008 \\
\hline
\end{tabular}

DSS - dextran sodium sulfate; TNBS - 2, 4, 6-trinitrobenzene sulfonic acid. 
human ulcerative colitis. ${ }^{31}$ After 6 days of 4\% DSS administration in Sprague-Dawley rats, Gaudio et al. reported focal erosions of the epithelium with slight crypt dilatation and lesions similar to the ones found in our study. ${ }^{29}$ Chen et al. developed a model of colitis in Sprague-Dawley rats using 2\% DSS for 3 days followed by an intracolonic administration of $30 \%$ ethanol, which produced severe ulceration and inflammation of the distal part of the rat colon, histologically characterized by increased specific infiltration, the presence of cryptic abscesses, and dysplasia. ${ }^{32}$

TNBS is a nitroaryl oxidizing acid with extreme oxidizing properties; when dissolved in ethanol, it induces severe

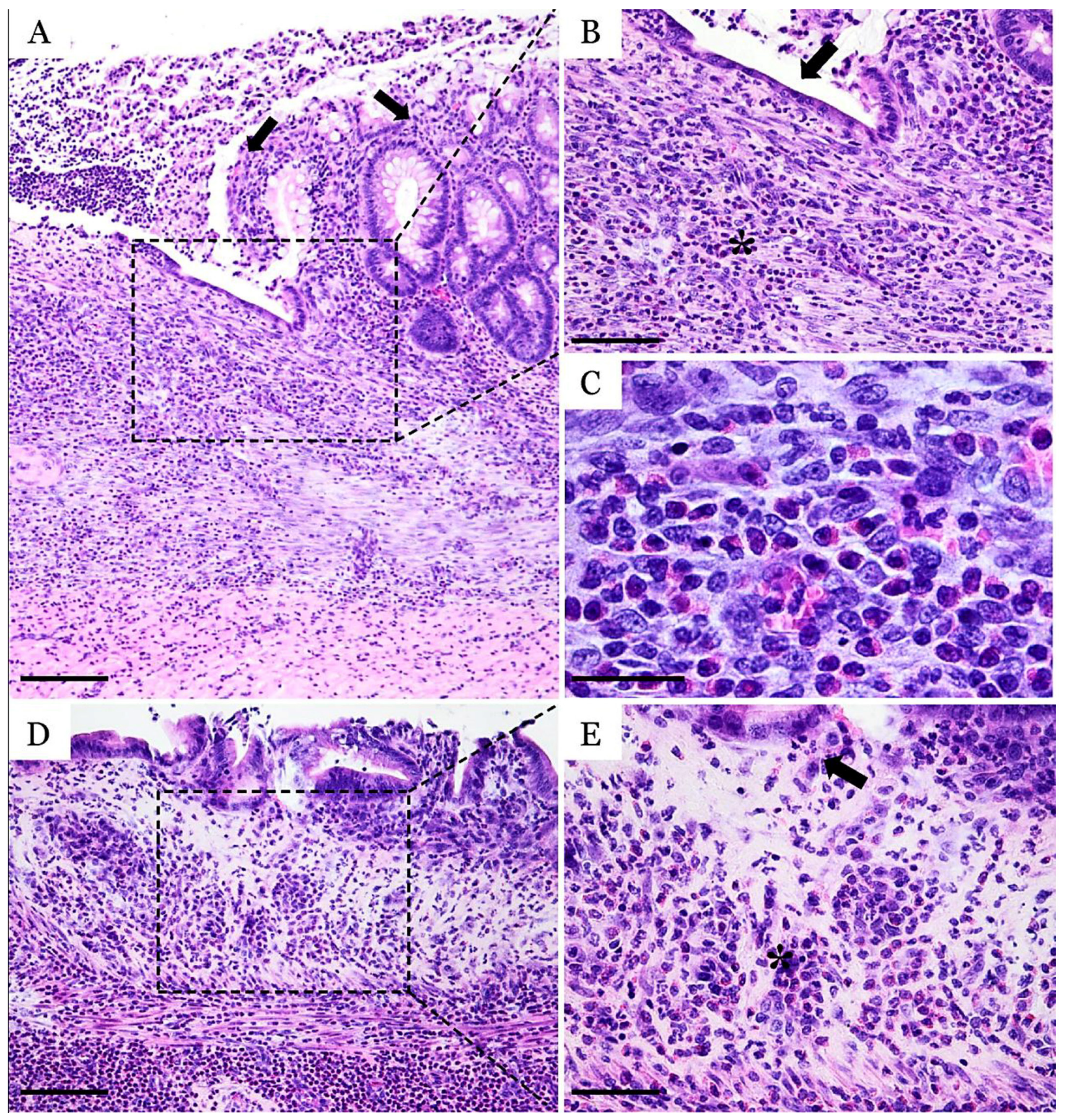

Fig. 3. Histopathological micrographs of TNBS-induced colitis

A - the margin of an ulcerative area involving the mucosa and submucosa of the colon; the massive inflammatory infiltrate extends through the muscularis layer. The adjacent mucosa present crypt dilation and distortion; B - detail of the delineated area from image A presenting the superficial part of the ulcer with epithelial regeneration (arrow), massive infiltration with PMN cells $\left(^{*}\right)$ and young, well-oriented granulation tissue; C - a high power view of the deep areas of the ulcer containing many PMN cells and rare mononuclear leukocytes; D - severe atrophy and fibrosis of the mucosa, with crypt distortion. Many polymorphonuclear cells are infiltrating the lamina propria, muscularis mucosa and submucosa; $\mathrm{E}$ - detail of the lamina propria from image $D$ presenting severe fibrosis and inflammatory infiltration with PMN cells (*) and rare mononuclear leukocytes (arrow); H\&E stain, OBX-10 for images A and D (scale bar $=400 \mu \mathrm{m}), \mathrm{OBX}-40$ for images $\mathrm{B}$ and $\mathrm{E}(\mathrm{scale}$ bar $=80 \mu \mathrm{m})$, OBX-100 for image C (scale bar $=50 \mu \mathrm{m})$. 
colonic necrosis surrounded by acute inflammation areas. TNBS reduces mucosal hydrophobicity by reacting with the surface-active phospholipids of the colonic mucosa, thus inducing colonic inflammation. ${ }^{1,33}$ Ethanol is very commonly used as a "barrier breaker" by increasing mucosal permeability. A failure in this barrier may result in intestinal inflammation, most likely through exposure to fecal antigens. ${ }^{32}$ In TNBS-induced colitis in rats, the inflammatory response includes mucosal and submucosal infiltration by lymphocytes, macrophages, polymorphonuclear leukocytes, connective tissue mast cells, and fibroblasts. ${ }^{34}$ The TNBS model highlights the fact that the loss of immune tolerance could lead to chronic intestinal inflammation. Moreover, modulation of the regulatory immune cells acts as a potential treatment method for the proper management of inflammatory intestinal disorders. ${ }^{35}$ Additionally, this colitis model served as a source of knowledge about the cytokine profile in human IBD and also as a way to treat the disease in humans. Moreover, it continues to be an advantageous platform for studying the fundamental aspects of human IBD in terms of its spontaneous fibrosis and resolution. ${ }^{1}$ The activation of the immune system leads to the increased production of proinflammatory cytokines, such as IL-17, TNF- $\alpha$, IL-1 $\beta$, IL-6, and IL-21, ROS, and prostaglandins contributing to a chronic inflammatory process. $^{15}$

In our study, the lesions produced by TNBS were characterized by a higher histological score for all criteria than the ones generated by DSS; in 4 of the 5 criteria, the histological scores were statistically significant. In the DSS group, the highest histological score $(1.6 \pm 0.89)$ was recorded for the criterion which described the extent of the ulcer, in most cases with a punctuate aspect. For the TNBS group, the highest histological score $(3.2 \pm 0.44)$ was recorded for the extent of inflammation, characterized mostly by mucosal/submucosal and muscle involvement. Significantly reduced colon length as a result of muscular contraction compared to controls was also reported by Gaudio et al. in rat experimental colitis after oral DSS administration. ${ }^{29}$

\section{Conclusions}

The experimental DSS- or TNBS-induced bowel inflammation used in this study corresponds to human IBD and is reproducible with characteristics indicative of acute inflammation in the case of the protocols mentioned.

\section{References}

1. Kiesler P, Fuss IJ, Strober W. Experimental models of inflammatory bowel diseases. Cell Mol Gastroenterol Hepatol. 2015;1(2):154-170.

2. Fiocchi C. Inflammatory bowel disease pathogenesis: Where are we? J Gastroenterol Hepatol. 2015;1:12-18.

3. Cătană CS, Berindan-Neagoe I, Cozma V, Magdaş C, Tăbăran F, Dumitraşcu DL. Contri-bution of the IL-17/IL-23 axis to the pathogenesis of inflammatory bowel disease. World J Gastroenterol. 2015;21(19):5823-5830.
4. McCracken JM, Jiang L, Deshpande KT, O'Neil MF, Pritchard MT. Differential effects of hyaluronan synthase 3 deficiency after acute vs chronic liver injury in mice. Fibrogenesis Tissue Repair. 2016;31(9):4. doi:10.1186/s13069-016-0041-5

5. Sugimoto MA, Vago JP, Teixeira MM, Sousa LP. Annexin A1 and the resolution of in-flammation: Modulation of neutrophil recruitment, apoptosis, and clearance. J Immunol Res. 2016;2016:8239258. doi:10.1155/2016/8239258

6. Hao LY, Liu X, Franchi L. Inflammasomes in inflammatory bowel disease pathogenesis. Curr Opin Gastroenterol. 2013;29:363-369.

7. Oh SY, Cho KA, Kang JL, Kim KH, Woo SY. Comparison of experimental mouse models of inflammatory bowel disease. Int J Mol Med. 2014;33(2):333-340.

8. Elson CO, Sartor RB, Tennyson GS, Riddell RH. Experimental models of inflammatory bowel disease. Gastroenterology. 1995;109:1344-1367.

9. Jiminez JA, Uwiera TC, Douglas Inglis G, Uwiera RR. Animal models to study acute and chronic intestinal inflammation in mammals. Gut Pathog. 2015;7:29. doi:10.1186/s13099-015-0076-y

10. Yan Y, Kolachala V, Dalmasso G, et al. Temporal and spatial analysis of clinical and molecular parameters in dextran sodium sulfate induced colitis. PLoS One. 2009;4(6):e6073.

11. Johansson ME, Gustafsson JK, Sjoberg KE, et al. Bacteria penetrate the inner mucus layer before inflammation in the dextran sulfate colitis model. PloS One. 2010;5(8):e12238. doi:10.1371/journal. pone.0012238

12. Laroui H, Ingersoll SA, Liu HC, et al. Dextran sodium sulfate (DSS) induces colitis in mice by forming nano-lipocomplexes with medium-chain-length fatty acids in the colon. PLoS One. 2012;7(3):e32084.

13. Chami B, Yeung AW, van Vreden C, King, NJ, Bao, B. The role of CXCR3 in DSS-induced colitis. PloS One. 2014;9(7):e101622. doi:10.1371/journal.pone.0101622

14. Alex P, Zachos NC, Nguyen T, et al. Distinct cytokine patterns identified from multiplex profiles of murine DSS and TNBS-induced colitis. Inflamm Bowel Dis. 2009;15(3):341-352. doi:10.1002/ibd.20753

15. Pandurangan AK, Ismail S, Saadatdoust Z, Esa NM. Allicin alleviates dextran sodium sulfate- (DSS-) induced ulcerative colitis in BALB/C mice. Oxid Med Cell Longev. 2015;2015:605208. doi:10.1155/2015/605208

16. Tham DM, Whitin JC, Cohen HJ. Increased expression of extracellular glutathione perox-idase in mice with dextran sodium sulfateinduced experimental colitis. Pediatr Res. 2002;51(5):641-646.

17. Randhawa PK, Singh K, Singh N, Jaggy AS. A review on chemicalinduced inflammatory bowel disease models in rodents. Korean JPhysiol Pharmacol. 2014;18(4):279-288. doi:10.4196/kjpp.2014.18.4.279

18. Barnett M, Fraser A. Animal models of colitis: Lessons learned, and their relevance to the clinic. In: $\mathrm{O}^{\prime}$ Connor $\mathrm{M}$, ed. Ulcerative Colitis - Treatments, Special Populations and the Future. Rijeka: InTech; 2011:173-178.

19. Tăbăran AF. Tehnici în histopatologie. In: Cătoi C, Gal A, Taulescu M. Tehnici de Anatomie Patologică Veterinară. Cluj-Napoca, Romania: Editura AcademicPres; 2014:136-153.

20. Rachmilewitz D, Karmeli F, Takabayashi K, et al. Immunostimulatory DNA ameliorates experimental and spontaneous murine colitis. Gastroenterology. 2002;122(5):1428-1441.

21. Paglia DE, Valentine WN. Studies on the quantitative and qualitative characterization of erythrocyte glutathione peroxidase. J Lab Clin Med. 1967;70(1):158-169.

22. Drabkin DL, Austin JH. Spectrophotometric studies: Spectrophotometric constants for common hemoglobin derivatives in human, dog and rabbit blood. J Biol Chem. 1932;98:719-733.

23. Balmus IM, Ciobica A, Trifan A, Stanciu C. The implications of oxidative stress and an-tioxidant therapies in inflammatory bowel disease: Clinical aspects and animal models. Saudi J Gastroenterol. 2016;22(1):3-17. doi:10.4103/1319-3767.173753

24. Iliev ID, Funari VA, Taylor KD, et al. Interactions between commensal fungi and the C-type lectin receptor Dectin-1 influence colitis. Science. 2012;336:1314-1317.

25. Wlodarska M, Thaiss CA, Nowarski R, Henao-Mejia J, Zhang JP, Brown EM. NLRP6 inflammasome orchestrates the colonic hostmicrobial interface by regulating goblet cell mucus secretion. Cell. 2014;156(5):1045-1059.

26. Sokol H, Conway KL, Zhang M, et al. Card9 mediates intestinal epithelial cell restitution, $T$-helper 17 responses, and control of bacterial infection in mice. Gastroenterology. 2013;145:591-601. 
27. Ren $G$, Sun A, Deng $C$, et al. The anti-inflammatory effect and potential mechanism of cardamonin in DSS- induced colitis. Am J Physiol Gastrointest Liver Physiol. 2015;309(7):G517-527. doi:10.1152/ ajpgi.00133.2015

28. Chae WJ, Ehrlich AK, Chan PY, et al. The Wnt antagonist Dickkopf-1 promotes pathological type 2 cell-mediated inflammation. Immunity. 2016;44(2):246-258. doi:10.1016/j.immuni.2016.01.008

29. Gaudio E, Taddei G, Vetuschi A, et al. Dextran sulfate sodium (DSS) colitis in rats: Clinical, structural, and ultrastructural aspects. Dig Dis Sci. 1999;44:1458-1475.

30. Rayudu V, Raju AB. Effect of Triphala on dextran sulphate sodiuminduced colitis in rats. Ayu. 2014;35(3):333-338.

31. Kishimoto S, Kobayashi H, Shimizu S, et al. Changes of colonic vasoactive intestinal peptide and cholinergic activity in rats with chemical colitis. Dig Dis Sci. 1992;37:1729-1737.

32. Chen Y, Si JM, Liu WL, et al. Induction of experimental acute ulcerative colitis in rats by administration of dextran sulfate sodium at low concentration followed by intracolonic ad-ministration of $30 \%$ ethanol. J Zhejiang Univ Sci B. 2007;8(9):632-637.

33. Tatsumi Y, Lichtenberger LM. Molecular association of trinitrobenzenesulfonic acid and surface phospholipids in the development of colitis in rats. Gastroenterology. 1996;110(3):780-789.

34. Coskun ZK, Kerem M, Gurbuz N, et al. The study of biochemical and histopathological effects of spirulina in rats with TNBS-induced colitis. Bratis/ Lek Listy. 2011;112(5):235-243.

35. Sun $\mathrm{M}, \mathrm{He} C$, Cong $\mathrm{Y}$, Liu Z. Regulatory immune cells in regulation of intestinal inflammatory response to microbiota. Mucosal Immunol. 2015;8(5):969-978. 